\title{
ON THE NAVIER BOUNDARY CONDITION FOR VISCOUS FLUIDS IN ROUGH DOMAINS
}

J. CASADO-DÍAZ*, M. LUNA-LAYNEZ* ${ }^{*}$, F.J. SUÁREZ-GRAU ${ }^{\dagger}$

* Departamento de Ecuaciones Diferenciales y Análisis Numérico, Universidad de Sevilla

${ }^{\dagger}$ Departamento de Matemáticas, Universidad de Huelva

jcasadod@us.es, mllaynez@us.es, fjsgrau@us.es

\begin{abstract}
In this paper we review some recent results concerning the study of the asymptotic behavior of viscous fluids in rough domains assuming Navier boundary conditions on the rough boundary. Our main interest is to study the relation between both the adherence and the Navier boundary conditions in the case of a boundary with weak rugosities. We show that the roughness acts on the fluid as a friction term. In particular, if the roughness is sufficiently strong, Navier condition implies adherence condition. This generalizes previous results of other authors.
\end{abstract}

Key words: Rough boundary; Stokes equations; Navier condition.

AMS subject classifications: $\quad 35 B 27,35 Q 30$

\section{Introduction}

A relevant problem in fluid mechanics is the appropriate choice of the boundary conditions. For a viscous fluid in an open set $\Omega \subset \mathbb{R}^{3}$, a well accepted hypothesis is that if the boundary is impermeable, then the fluid adheres completely to it. Denoting by $u$ the velocity of the fluid in $\Omega$, this adherence condition becomes

$$
u=0 \quad \text { on } \partial \Omega .
$$

However, some other boundary conditions are often used. In this sense, for a viscous fluid governed by the Stokes or Navier-Stokes system (with viscosity coefficient equals one), Navier proposed the slip-friction boundary condition, see [22]:

$$
u \cdot \nu=0, \quad T\left(\frac{\partial u}{\partial \nu}-p \nu+\gamma u\right)=0 \quad \text { on } \partial \Omega,
$$

where $p$ is the pressure, $\nu$ is the unitary outside normal vector to $\Omega$ on $\partial \Omega, T$ is the orthogonal projection on the tangent space to $\partial \Omega$ and $\gamma$ is a nonnegative constant. In (2) we are assuming that the boundary is impermeable (so the normal component

Received: March 27, 2011. Accepted: September 27, 2011. 
of the velocity, $u \cdot \nu$, vanishes on $\partial \Omega$ ), and that it exerts to the fluid a friction force opposite and proportional to the velocity. Writing the equilibrium forces equation on the boundary, but only in the tangential components, and denoting by $\gamma$ the friction coefficient, this gives the second equation in (2).

Taking into account that $p \nu$ is orthogonal to the tangent space to $\partial \Omega$, the Navier boundary condition can be also written as

$$
u \cdot \nu=0, \quad T\left(\frac{\partial u}{\partial \nu}+\gamma u\right)=0 \quad \text { on } \partial \Omega .
$$

Due to the freedom of choice of boundary conditions, a natural question is if there is any relationship between conditions (1) and (3). In this sense, it was considered in [10] a three-dimensional domain $\Omega_{\varepsilon}$ with a rough boundary described by the equation (see Figure 1)

$$
x_{3}=-\varepsilon \Psi\left(\frac{x^{\prime}}{\varepsilon}\right), \quad \forall x^{\prime} \in \omega,
$$

(along this paper a point $x \in \mathbb{R}^{3}$ is decomposed as $x=\left(x^{\prime}, x_{3}\right)$ with $x^{\prime} \in \mathbb{R}^{2}, x_{3} \in \mathbb{R}$ ) with $\omega$ a bounded open set of $\mathbb{R}^{2}$ and $\Psi$ a smooth periodic function such that

$$
\operatorname{Span}\left(\left\{\nabla \Psi\left(z^{\prime}\right): z^{\prime} \in \mathbb{R}^{2}\right\}\right)=\mathbb{R}^{2} .
$$

It was proved that if $u_{\varepsilon}$ is bounded in energy and satisfies $u_{\varepsilon} \cdot \nu=0$ on the boundary described by (4), then the weak limit $u$ of $u_{\varepsilon}$ vanishes on $\omega \times\{0\}$. So, in this case, the Navier and adherence conditions are asymptotically equivalent. This means that the adherence condition, which is experimentally observed, may be due to the existence of microrugosities.

Generalizations of this result have been obtained in [3] for a non-periodic boundary described by

$$
x_{3}=\Phi_{\varepsilon}\left(x^{\prime}\right), \quad \forall x^{\prime} \in \omega,
$$

where $\Phi_{\varepsilon}$ converges weakly-* to zero in $W^{1, \infty}(\omega)$ and it is such that the support of the Young's measure associated to $\nabla \Phi_{\varepsilon}$ contains two linearly independent vectors. Remark that this last condition implies that $\nabla \Phi_{\varepsilon}$ does not converge to zero in $L^{1}(\omega)^{2}$.

Our main goal in the present paper is to study the relation between the Navier and the adherence boundary conditions in the case of weak rugosities. The article, which is a review of the results which appear in the Ph.D. Thesis of F.J. Suárez-Grau (see [24]), is organized as follows

In Section 2, we study the asymptotic behavior of viscous fluids in the open set $\Omega_{\varepsilon}$ described by (see Figure 2)

$$
\Omega_{\varepsilon}=\left\{x=\left(x^{\prime}, x_{3}\right) \in \omega \times \mathbb{R}:-\delta_{\varepsilon} \Psi\left(\frac{x^{\prime}}{\varepsilon}\right)<x_{3}<1\right\},
$$

where $\omega \subset \mathbb{R}^{2}$ is a Lipschitz bounded open set, $\Psi \in W_{\text {loc }}^{2, \infty}\left(\mathbb{R}^{2}\right)$ is periodic of period $Z^{\prime}=(0,1)^{2}$, and $\delta_{\varepsilon}>0$ satisfies $\lim _{\varepsilon \rightarrow 0} \frac{\delta_{\varepsilon}}{\varepsilon}=0$. 
We impose the Navier condition on the oscillating boundary $\Gamma_{\varepsilon}$ of period $\varepsilon$ and amplitude $\delta_{\varepsilon}$ (with $\delta_{\varepsilon} \ll \varepsilon$ ) given by (see Figure 2)

$$
\Gamma_{\varepsilon}=\left\{x=\left(x^{\prime}, x_{3}\right) \in \omega \times \mathbb{R}: x_{3}=-\delta_{\varepsilon} \Psi\left(\frac{x^{\prime}}{\varepsilon}\right)\right\},
$$

and, to simplify, the adherence condition on the rest of the boundary $\partial \Omega_{\varepsilon} \backslash \Gamma_{\varepsilon}$.

Remark that in our case $\Phi_{\varepsilon}=\delta_{\varepsilon} \Psi\left(\frac{x^{\prime}}{\varepsilon}\right)$ converges strongly to zero in $W^{1, \infty}(\omega)$ and therefore the results in [3] do not apply.

Denoting by

$$
\lambda=\lim _{\varepsilon \rightarrow 0} \frac{\delta_{\varepsilon}}{\varepsilon^{\frac{3}{2}}} \in[0,+\infty],
$$

(the limit exists at least for a subsequence) we show

- If $\lambda=+\infty$ and (5) holds, then the Navier and adherence boundary conditions are asymptotically equivalent. This extends the result obtained in [10] for $\delta_{\varepsilon}=\varepsilon$ to the case when $\delta_{\varepsilon} / \varepsilon$ tends to zero and $\delta_{\varepsilon} / \varepsilon^{\frac{3}{2}}$ tends to infinity.

- If $\lambda=0$, the roughness is so small that it has no effect on the limit problem.

- If $\lambda \in(0,+\infty)$, the roughness is not strong enough to obtain the adherence condition, but it is large enough to make appear a new friction term. Namely, we obtain the following Navier boundary condition in the limit,

$$
u_{3}=0, \quad-\partial_{3} u^{\prime}+\gamma u^{\prime}+\lambda^{2} R u^{\prime}=0, \quad \text { on } \omega \times\{0\},
$$

where $R \in \mathbb{R}^{2 \times 2}$ is a symmetric and nonnegative matrix. The new term $\lambda^{2} R$ is similar to the strange term obtained by D. Cioranescu and F. Murat in [17] for the homogenization of Dirichlet problems in perforated domains. This case can be considered as the general case, because it provides the other two ones tending $\lambda$ to infinity or zero.

Related to this result, it has been studied in [6] the asymptotic behavior of viscous fluids confined in general rough domains, not necessarily periodic. In the particular case of a domain with a rough bottom described by

$$
x_{3}=\Psi_{\varepsilon}\left(x^{\prime}\right), \quad \forall x^{\prime} \in \omega,
$$

with $\Psi_{\varepsilon}$ converging weakly-* to zero in $W^{1, \infty}(\omega)$, the results in [6] imply that the limit boundary condition is

$$
u_{3}=0, \quad-\partial_{3} u^{\prime}+\gamma u^{\prime}+H u^{\prime} \mu=0, \quad \text { on } \omega \times\{0\},
$$

where $\mu$ is a nonnegative Borel measure, which can be infinity in compact sets of $\omega$, and $H$ is a $\mu$-measurable matrix evaluated function. Our results provide an example where the extra term $H u^{\prime} \mu$ is not zero. Another example of different nature for a ribbed boundary described by $x_{3}=\varepsilon \Psi\left(\frac{x_{1}}{\varepsilon}\right)$ is given in [4] and [5]. 
In Section 3 we consider the case of a thin domain $\Omega_{\varepsilon}^{\text {thin }}$ of small height $h_{\varepsilon}$ tending to zero described by

$$
\Omega_{\varepsilon}^{\text {thin }}=\left\{x=\left(x^{\prime}, x_{3}\right) \in \omega \times \mathbb{R}:-\delta_{\varepsilon} \Psi\left(\frac{x^{\prime}}{\varepsilon}\right)<x_{3}<h_{\varepsilon}\right\},
$$

with $\omega$ and $\Psi$ as above, and the parameters $h_{\varepsilon}$ and $\delta_{\varepsilon}$ satisfying

$$
\lim _{\varepsilon \rightarrow 0} \frac{\varepsilon}{h_{\varepsilon}}=0, \quad \lim _{\varepsilon \rightarrow 0} \frac{\delta_{\varepsilon}}{\varepsilon}=0 .
$$

We obtain a Reynolds system in the limit which shows that near the rough bottom $\Gamma_{\varepsilon}$ the behavior of the fluid is similar to the one obtained in Section 2 for fluids confined in domains of height one but with $\lambda$ replaced by

$$
\lambda_{t h i n}=\lim _{\varepsilon \rightarrow 0} \frac{\delta_{\varepsilon}}{\varepsilon^{\frac{3}{2}}} h_{\varepsilon}^{\frac{1}{2}} .
$$

Remark that $\lambda=\lambda_{\text {thin }}$ if $h_{\varepsilon}=1$.

The results obtained in Sections 2 and 3 show that the Navier boundary condition, which can be also written as

$$
u_{\varepsilon}(x) \in T_{\varepsilon}(x), \quad \frac{\partial u_{\varepsilon}}{\partial \nu}(x)+\gamma u_{\varepsilon}(x) \in T_{\varepsilon}(x)^{\perp}, \quad \text { on } \Gamma_{\varepsilon},
$$

with $T_{\varepsilon}(x)$ the tangent space in the point $x \in \Gamma_{\varepsilon}$, provides a new term in the limit equation. In Section 4 we study this phenomena in a more general setting. Instead of the Stokes or Navier-Stokes system, we consider a sequence of linear elliptic systems of $M$ equations posed in varying open sets $\Omega_{\varepsilon} \subset \mathbb{R}^{N}$, not necessarily periodic, with a boundary condition similar to (12), where $T_{\varepsilon}(x)$ is replaced by an arbitrary linear space $V_{\varepsilon}(x) \subset \mathbb{R}^{M}$. This abstract formulation contains a lot of classical boundary conditions. For instance it allows us to study the asymptotic behavior of linear elliptic systems in rough domains $\Omega_{\varepsilon}$ where we impose Dirichlet and Neumann boundary conditions on varying subsets of $\partial \Omega_{\varepsilon}$. This problem has been studied in [7] and [8] for $\Omega_{\varepsilon}=\Omega$ fixed.

The results of Section 4 could be extended to viscous fluids. For the particular choice $V_{\varepsilon}(x)=T_{\varepsilon}(x)$, it would recover the results in [6].

To finish this introduction, we refer some open problems in which we are starting working and we hope provide results in the near future:

- Extension to non-Newtonian viscous fluids, which are involved in Biology.

- Behavior of fluids in thin rough domains described by (9), assuming different behaviors for the parameters from the imposed in (10).

- Problems with free boundaries and applications to lubrication and Oceanography. 


\section{Asymptotic behavior of viscous fluids in rough domains with fixed height}

Given a Lipschitz bounded connected open set $\omega \subset \mathbb{R}^{2}$ and a function $\Psi \in W_{\text {loc }}^{2, \infty}\left(\mathbb{R}^{2}\right)$, periodic of period $Z^{\prime}=(0,1)^{2}$, we define the domain $\Omega_{\varepsilon}$ by (6) and the rough portion of the boundary $\Gamma_{\varepsilon}$ by (7). We also define $\Omega=\omega \times(0,1)$ and $\Gamma=\omega \times\{0\}$.

In $\Omega_{\varepsilon}$, we consider the solution $\left(u_{\varepsilon}, p_{\varepsilon}\right)$ of the following Stokes system satisfying the Navier condition on the rough boundary $\Gamma_{\varepsilon}$ and the adherence condition on the rest of the boundary $\partial \Omega_{\varepsilon} \backslash \Gamma_{\varepsilon}$,

$$
\left\{\begin{array}{l}
-\Delta u_{\varepsilon}+\nabla p_{\varepsilon}=f \text { in } \Omega_{\varepsilon}, \quad \operatorname{div} u_{\varepsilon}=0 \text { in } \Omega_{\varepsilon} \\
u_{\varepsilon} \cdot \nu=0 \text { on } \Gamma_{\varepsilon}, \quad T_{\varepsilon}\left(\frac{\partial u_{\varepsilon}}{\partial \nu}+\gamma u_{\varepsilon}\right)=0 \text { on } \Gamma_{\varepsilon} \\
u_{\varepsilon}=0 \quad \text { on } \partial \Omega_{\varepsilon} \backslash \Gamma_{\varepsilon},
\end{array}\right.
$$

where $\gamma \geq 0$ is a friction coefficient, $\nu$ denotes the unitary outside normal vector to $\Omega_{\varepsilon}$ on $\Gamma_{\varepsilon}, T_{\varepsilon}$ is the orthogonal projection on the tangent space to $\Gamma_{\varepsilon}$, and the second member $f$ is in $L^{2}(\omega \times \mathbb{R})^{3}$ (more general second members can be considered).

The system (13) has a unique solution $\left(u_{\varepsilon}, p_{\varepsilon}\right) \in H^{1}\left(\Omega_{\varepsilon}\right)^{3} \times L_{0}^{2}\left(\Omega_{\varepsilon}\right)\left(L_{0}^{2}\left(\Omega_{\varepsilon}\right)\right.$ denotes the space of functions in $L^{2}\left(\Omega_{\varepsilon}\right)$ whose integral in $\Omega_{\varepsilon}$ is zero). Moreover, we prove that there exists $C>0$ such that

$$
\left\|u_{\varepsilon}\right\|_{H^{1}\left(\Omega_{\varepsilon}\right)^{3}}+\left\|p_{\varepsilon}\right\|_{L^{2}\left(\Omega_{\varepsilon}\right)} \leq C, \quad \forall \varepsilon>0 .
$$

Our problem is to describe the asymptotic behavior of the sequences $u_{\varepsilon}$ and $p_{\varepsilon}$. This is given by the following theorem which is the main result of this section.

Theorem 1 The solution $\left(u_{\varepsilon}, p_{\varepsilon}\right)$ of (13) satisfies

$$
u_{\varepsilon} \rightarrow u \text { in } H^{1}(\Omega)^{3}, \quad p_{\varepsilon} \rightarrow p \text { in } L^{2}(\Omega),
$$

with $(u, p)$ the unique solution of

$$
\left\{\begin{array}{l}
-\Delta u+\nabla p=f \text { in } \Omega, \quad \operatorname{div} \mathrm{u}=0 \text { in } \Omega \\
u_{3}=0 \text { on } \Gamma, \quad u=0 \text { on } \partial \Omega \backslash \Gamma,
\end{array}\right.
$$

plus a boundary condition for $u^{\prime}$ which depends on the parameter $\lambda$ defined by (8). More precisely we have

i) If $\lambda=0$, then

$$
-\partial_{3} u^{\prime}+\gamma u^{\prime}=0 \text { on } \Gamma .
$$

ii) If $\lambda \in(0,+\infty)$, then defining $\left(\widehat{\phi}^{i}, \widehat{q}^{i}\right), i=1,2$, as the solution of

$$
\left\{\begin{array}{l}
-\Delta_{z} \widehat{\phi}^{i}+\nabla_{z} \widehat{q}^{i}=0, \quad \operatorname{div}_{z} \widehat{\phi}^{i}=0 \text { in } \mathbb{R}^{2} \times(0,+\infty) \\
\widehat{\phi}_{3}^{i}\left(z^{\prime}, 0\right)+\partial_{z_{i}} \Psi\left(z^{\prime}\right)=0, \partial_{z_{3}}\left(\widehat{\phi}^{i}\right)^{\prime}\left(z^{\prime}, 0\right)=0, \quad \text { a.e. } z^{\prime} \in \mathbb{R}^{2} \\
\widehat{\phi}^{i}\left(., z_{3}\right), \widehat{q}^{i}\left(., z_{3}\right) \text { periodic of period } Z^{\prime}, \quad \text { a.e. } z_{3} \in(0,+\infty) \\
\widehat{\phi}^{i} \in H^{1}\left(Z^{\prime} \times(0,+\infty)\right)^{3}, \widehat{q}^{i} \in L^{2}\left(Z^{\prime} \times(0,+\infty)\right),
\end{array}\right.
$$


and $R \in \mathbb{R}^{2 \times 2}$ by

$$
R_{i j}=\int_{Z^{\prime} \times(0,+\infty)} D_{z} \widehat{\phi}^{i}: D_{z} \widehat{\phi}^{j} d z, \quad \forall i, j \in\{1,2\}
$$

we have

$$
-\partial_{3} u^{\prime}+\gamma u^{\prime}+\lambda^{2} R u^{\prime}=0 \text { on } \Gamma .
$$

iii) If $\lambda=+\infty$, then defining

$$
W=\operatorname{Span}\left(\left\{\nabla \Psi\left(z^{\prime}\right): z^{\prime} \in Z^{\prime}\right\}\right),
$$

we have

$$
u^{\prime} \in W^{\perp} \text { on } \Gamma, \quad-\partial_{3} u^{\prime}+\gamma u^{\prime} \in W, \text { on } \Gamma
$$

Remark 1 For $\lambda=0$, Theorem 1 shows that the roughness of $\Gamma_{\varepsilon}$ is very slight and so the solution $\left(u_{\varepsilon}, p_{\varepsilon}\right)$ of (13) behaves as if $\Gamma_{\varepsilon}$ coincides with the plane boundary $\Gamma$. For $\lambda \in(0,+\infty)$ (critical size), the boundary condition satisfied by the limit $u$ of $u_{\varepsilon}$ on the tangent space to $\Gamma$ contains the new term $\lambda^{2} R u^{\prime}$. In this case, the effect of the roughness of $\Gamma_{\varepsilon}$ is not worthless and it makes to appear this new term in the limit. Finally, for $\lambda=+\infty$ the roughness of $\Gamma_{\varepsilon}$ is so strong that the limit $u$ of $u_{\varepsilon}$ does not only satisfies the condition $u_{3}=0$ on $\Gamma$, but also its tangent velocity on $\Gamma, u^{\prime}$, is orthogonal to the vectors $\nabla \Psi\left(z^{\prime}\right)$, with $z^{\prime} \in Z^{\prime}$. In particular, if the space $W$ defined by (19) has dimension 2, then $u$ satisfies the adherence condition $u=0$ on $\Gamma$. This extends to the case where

$$
\lim _{\varepsilon \rightarrow 0} \frac{\delta_{\varepsilon}}{\varepsilon}=0, \quad \lim _{\varepsilon \rightarrow 0} \frac{\delta_{\varepsilon}}{\varepsilon^{\frac{3}{2}}}=+\infty,
$$

the results obtained in [10] for $\delta_{\varepsilon}=\varepsilon$.

Remark 2 The case $\lambda \in(0,+\infty)$ can be considered as the general one. In fact, if $\lambda$ tends to zero or infinity in (18) we get (15) or (20) respectively.

Sketch of the proof of Theorem 1 Since $\left(u_{\varepsilon}, p_{\varepsilon}\right)$ is bounded in $H^{1}\left(\Omega_{\varepsilon}\right)^{3} \times L^{2}\left(\Omega_{\varepsilon}\right)$ it is clear that (at least for a subsequence) it converges weakly in $H^{1}(\Omega)^{3} \times L^{2}(\Omega)$ to some $(u, p)$ which is a solution of the Stokes system (14). The difficulty is then to obtain the boundary condition in the tangent space to $\Gamma$. For this purpose we need to study more carefully the behavior of $u_{\varepsilon}$ near $\Gamma_{\varepsilon}$. This is carried out using an original adaptation of the unfolding method, [2], [9], [16], which is very related to the twoscale convergence method, [1], [21], [23]. The idea is to introduce suitable changes of variables which transform every periodic cell into a simpler reference set by using a supplementary variable (microscopic variable). In our case, given $\left(u_{\varepsilon}, p_{\varepsilon}\right)$ solution of (13), and defining

$$
\hat{\omega}_{\varepsilon}=\bigcup_{\substack{k^{\prime} \in \mathbb{Z}^{2} \\ \varepsilon k^{\prime}+\varepsilon Z^{\prime} \subset \omega}}\left(\varepsilon k^{\prime}+\varepsilon Z^{\prime}\right), \quad K_{\varepsilon}=\left\{z \in Z^{\prime} \times \mathbb{R}:-\frac{\delta_{\varepsilon}}{\varepsilon} \Psi\left(z^{\prime}\right)<z_{3}<\frac{1}{\varepsilon}\right\},
$$


we introduce $\hat{u}_{\varepsilon}: \hat{\omega}_{\varepsilon} \times K_{\varepsilon} \rightarrow \mathbb{R}^{3}, \hat{p}_{\varepsilon}: \hat{\omega}_{\varepsilon} \times K_{\varepsilon} \rightarrow \mathbb{R}$ by

$$
\hat{u}_{\varepsilon}\left(x^{\prime}, z\right)=\sum_{\substack{k^{\prime} \in \mathbb{Z}^{\prime} \\ \varepsilon k^{\prime}+\varepsilon Z^{\prime} \subset \omega}} u_{\varepsilon}\left(\varepsilon k^{\prime}+\varepsilon z^{\prime}, \varepsilon z_{3}\right) \chi_{\varepsilon k^{\prime}+\varepsilon Z^{\prime}}\left(x^{\prime}\right) .
$$

Observe that $\hat{u}_{\varepsilon}$ is obtained transforming every column $\Omega_{\varepsilon} \cap\left(\left(\varepsilon k^{\prime}+\varepsilon Z^{\prime}\right) \times \mathbb{R}\right)$ in the set $K_{\varepsilon}$ by using the change of variables $z=\left(\left(x^{\prime}-\varepsilon k^{\prime}\right) / \varepsilon, x_{3} / \varepsilon\right)$. Here, $x^{\prime}$ is the macroscopic variable and $z$ the microscopic one. Moreover, the set $K_{\varepsilon}$ converges to the set $Z^{\prime} \times(0,+\infty)$, while $\left(\varepsilon k^{\prime}+\varepsilon Z^{\prime}\right) \times \mathbb{R}$ converges to the empty set. The asymptotic behavior of $u_{\varepsilon}$ near $\Gamma_{\varepsilon}$ is obtained by studying the asymptotic behavior of $\hat{u}_{\varepsilon}$. Namely, from $u_{\varepsilon}$ bounded in $H^{1}\left(\Omega_{\varepsilon}\right)^{3}$, we deduce

$$
\frac{1}{\varepsilon} \int_{\hat{\omega}_{\varepsilon} \times K_{\varepsilon}}\left|D_{z} \hat{u}_{\varepsilon}\right|^{2} d x^{\prime} d z \leq C
$$

and thus (for a subsequence)

$$
\hat{w}_{\varepsilon}\left(x^{\prime}, z\right)=\frac{1}{\sqrt{\varepsilon}}\left(\hat{u}_{\varepsilon}\left(x^{\prime}, z\right)-\int_{Z^{\prime}} \hat{u}_{\varepsilon}\left(x^{\prime}, \rho^{\prime}, 0\right) d \rho^{\prime}\right) \rightarrow \hat{w}
$$

in $L^{2}\left(\omega ; H^{1}\left(Z^{\prime} \times(0, M)\right)^{3}\right)$, for every $M>0$, with $\hat{w} \in L^{2}\left(\omega ; H^{1}\left(Z^{\prime} \times(0, M)\right)^{3}\right)$, $D_{z} \hat{w} \in L^{2}\left(\omega ; L^{2}\left(Z^{\prime} \times(0,+\infty)\right)^{3 \times 3}\right)$. Moreover, we can prove that $\hat{u}$ is periodic with respect to $z^{\prime}$.

Observing that

$$
\int_{Z^{\prime}} \hat{u}_{\varepsilon}\left(x^{\prime}, \rho^{\prime}, 0\right) d \rho^{\prime}=\frac{1}{\varepsilon^{2}} \int_{\varepsilon k^{\prime}+\varepsilon Z^{\prime}} u_{\varepsilon}\left(y^{\prime}, 0\right) d y^{\prime}, \quad x^{\prime} \in \varepsilon k^{\prime}+\varepsilon Z^{\prime},
$$

we also deduce from (21) that

$$
\hat{u}_{\varepsilon}\left(x^{\prime}, z\right) \rightarrow u \text { in } L^{2}\left(\omega ; H^{1}\left(Z^{\prime} \times(0, M)\right)^{3}\right), \quad \forall M>0 .
$$

On the other hand, the condition $u_{\varepsilon} \cdot \nu=0$ on $\Gamma_{\varepsilon}$ allows us to show

$$
\frac{\delta_{\varepsilon}}{\varepsilon^{\frac{3}{2}}} \nabla \Psi\left(z^{\prime}\right) \cdot \hat{u}_{\varepsilon}^{\prime}\left(x^{\prime}, z^{\prime}, 0\right)+\hat{w}_{\varepsilon, 3}\left(x^{\prime}, z^{\prime}, 0\right) \rightarrow 0 \text { in } L^{2}\left(\omega \times Z^{\prime}\right) .
$$

In order to obtain the boundary condition for the limit system in the tangent space to $\Gamma$ the reasoning depends on the limit $\lambda$ of $\delta_{\varepsilon} / \varepsilon^{\frac{3}{2}}$.

- If $\lambda=+\infty$, then (23) and (22) prove that $u^{\prime}$ belongs to $W^{\perp}$ a.e. on $\Gamma$, which gives the first assertion in (20). The second one is obtained using test functions $v$ in (13) which a.e. in $\Gamma$ satisfy $v_{3}=0, v^{\prime} \in W^{\perp}$.

- If $\lambda \in(0,+\infty)$, passing to the limit in (23) we deduce

$$
\lambda \nabla \Psi\left(z^{\prime}\right) \cdot u^{\prime}\left(x^{\prime}, 0\right)+\hat{w}_{3}\left(x^{\prime}, z^{\prime}, 0\right)=0 .
$$

On the other hand, (21) and $\hat{u}$ periodic in $z^{\prime}$ suggest that $u_{\varepsilon}(x)$ behaves as $u(x)+$ $\sqrt{\varepsilon} \hat{w}\left(x^{\prime}, \frac{x}{\varepsilon}\right)$. The proof of (18) is obtained using test functions in (13) of the form $v_{\varepsilon}(x)=v(x)+\sqrt{\varepsilon} \hat{v}\left(x^{\prime}, x / \varepsilon\right)$ (slightly modified to have $v_{\varepsilon} \cdot \nu=0$ on $\Gamma_{\varepsilon}$ ) with $v^{\prime}=0$ 
on $\partial \Omega \backslash \Gamma, v_{3}=0$ on $\partial \Omega, \hat{v}$ periodic in $z^{\prime}, \hat{v}\left(x^{\prime}, z\right)=0$ if $z_{3}$ is large, and satisfying $\hat{v}_{3}\left(x^{\prime}, z^{\prime}, 0\right)=-\lambda v^{\prime}\left(x^{\prime}, 0\right)$. In particular this proves the equality

$$
\hat{w}\left(x^{\prime}, z\right)=u_{1}\left(x^{\prime}, 0\right) \widehat{\Phi}^{1}(z)+u_{2}\left(x^{\prime}, 0\right) \widehat{\Phi}^{2}(z), \text { a.e. in } \omega \times Z^{\prime} \times(0,+\infty) .
$$

- If $\lambda=0$, we consider $v$ as above, such that $v^{\prime}=0$ on $\partial \Omega \backslash \Gamma, v_{3}=0$ on $\partial \Omega$ and then, we use as test function

$$
v_{\varepsilon}(x)=\left(v^{\prime}(x), v_{3}(x)-\frac{\delta_{\varepsilon}}{\varepsilon} \zeta\left(\frac{x_{3}}{\varepsilon}\right) \nabla \Psi\left(\frac{x^{\prime}}{\varepsilon}\right) \cdot v^{\prime}\left(x^{\prime}\right)\right),
$$

with $\zeta$ a smooth function such that $\zeta(t)=0$ in $(-\infty, 0), \zeta(t)=1$ in $(1,+\infty)$.

Theorem 1 gives an approximation of $\left(u_{\varepsilon}, p_{\varepsilon}\right)$ in the weak topology of $H^{1}(\Omega)^{3} \times$ $L^{2}(\Omega)$. Indeed, we have the following result relative to the strong convergence of $\left(D u_{\varepsilon} \chi_{\Omega_{\varepsilon}}, p_{\varepsilon} \chi_{\Omega_{\varepsilon}}\right)$ in $H^{1}(\Omega)^{3} \times L^{2}(\Omega)$ (corrector result).

Theorem 2 Under the assumptions of Theorem 1 we have

i) If $\lambda=0$ or $+\infty$, then

$$
\lim _{\varepsilon \rightarrow 0}\left(\left\|u_{\varepsilon}\right\|_{H^{1}\left(\Omega_{\varepsilon} \backslash \Omega\right)^{3}}+\left\|p_{\varepsilon}\right\|_{L^{2}\left(\Omega_{\varepsilon} \backslash \Omega\right)}+\left\|u_{\varepsilon}-u\right\|_{H^{1}(\Omega)^{3}}+\left\|p_{\varepsilon}-p\right\|_{L^{2}(\Omega)}\right)=0 .
$$

ii) If $\lambda \in(0,+\infty)$, then, taking $\left(\widehat{\phi}^{i}, \widehat{q}^{i}\right), i=1,2$, as the solution of (16), and defining $u^{b}$ and $p^{b}$ by

$$
\begin{aligned}
& u^{b}(x, z)=u_{1}(x) \widehat{\phi}^{1}(z)+u_{2}(x) \widehat{\phi}^{2}(z), \\
& p^{b}(x, z)=u_{1}(x) \widehat{q}^{1}(z)+u_{2}(x) \widehat{q}^{2}(z),
\end{aligned}
$$

we have

$$
\begin{gathered}
\lim _{\varepsilon \rightarrow 0}\left(\left\|u_{\varepsilon}\right\|_{H^{1}\left(\Omega_{\varepsilon} \backslash \Omega\right)^{3}}+\left\|u_{\varepsilon}-u\right\|_{L^{2}(\Omega)}+\left\|D u_{\varepsilon}-D u-\frac{\lambda}{\sqrt{\varepsilon}} D_{z} u^{b}\left(x, \frac{x}{\varepsilon}\right)\right\|_{L^{2}(\Omega)^{3 \times 3}}\right)=0, \\
\lim _{\varepsilon \rightarrow 0}\left(\left\|p_{\varepsilon}\right\|_{L^{2}\left(\Omega_{\varepsilon} \backslash \Omega\right)}+\left\|p_{\varepsilon}-p-\frac{\lambda}{\sqrt{\varepsilon}} p^{b}\left(x, \frac{x}{\varepsilon}\right)\right\|_{L^{2}(\Omega)}\right)=0 .
\end{gathered}
$$

This corrector result can be improved obtaining an estimate for the difference of $\left(u_{\varepsilon}, p_{\varepsilon}\right)$ and its corrector. We focus in the case $\lambda \in(0,+\infty)$ which, as we said in Remark 2, can be considered as the general one. Assuming $\delta_{\varepsilon}=\lambda \varepsilon^{\frac{3}{2}}$, with $\lambda \in(0,+\infty)$, we prove the following theorem

Theorem 3 If the function $u$ defined by (14) and (18) belongs to $H^{s}(\Omega)^{3}$, with $s>3 / 2$, then we have

$$
\begin{gathered}
\left\|u_{\varepsilon}\right\|_{H^{1}\left(\Omega_{\varepsilon} \backslash \Omega\right)^{3}}+\left\|u_{\varepsilon}-u-\lambda \sqrt{\varepsilon} u^{b}\left(x, \frac{x}{\varepsilon}\right)\right\|_{H^{1}(\Omega)^{3}} \leq C \sqrt{\varepsilon}, \\
\left\|p_{\varepsilon}\right\|_{L^{2}\left(\Omega_{\varepsilon} \backslash \Omega\right)}+\left\|p_{\varepsilon}-p-\frac{\lambda}{\sqrt{\varepsilon}} p^{b}\left(x, \frac{x}{\varepsilon}\right)\right\|_{L^{2}(\Omega)} \leq C \sqrt{\varepsilon} .
\end{gathered}
$$


Sketch of the proof. The proof consists in showing that the pair $\left(\tilde{u}_{\varepsilon}, \tilde{p}_{\varepsilon}\right)=(u+$ $\left.\lambda \sqrt{\varepsilon} u^{b}\left(x, \frac{x}{\varepsilon}\right), p+\frac{\lambda}{\sqrt{\varepsilon}} p^{b}\left(x, \frac{x}{\varepsilon}\right)\right)$ satisfies a Stokes system with right-hand side and boundary conditions close to the ones satisfied by $\left(u_{\varepsilon}, p_{\varepsilon}\right)$. Then, usual estimates for the Stokes problem applied to the difference of the equations satisfied by $\left(u_{\varepsilon}, p_{\varepsilon}\right)$ and $\left(\tilde{u}_{\varepsilon}, \tilde{p}_{\varepsilon}\right)$ give the result.

In order to apply Theorem 3, we need the solution $u$ of (14) and (18) in $H^{s}(\Omega)^{3}$, $s \geq 3 / 2$. A result in this sense is given by the following proposition.

Proposition 4 The solution (u,p) of (14)-(18) is in $H^{2}(O \times(0,1))^{3} \times H^{1}(O \times(0,1))$, for every open set $O$ completely contained in $\omega$.

Sketch of the proof. Since $u^{\prime}$ belongs to $H^{\frac{1}{2}}(\Gamma)^{2}$, there exists (see e.g. [20]) $z^{\prime}: \omega \times(0,1) \rightarrow \mathbb{R}^{2}$, with $z^{\prime} \in H^{2}(O \times(0,1))^{2}$, for every open set $O$ completely contained in $\omega$, such that $z^{\prime}=0$ on $\omega \times\{1\}, \partial_{3} z^{\prime}=\lambda^{2} R u^{\prime}$ on $\Gamma$. Then, defining $v=\left(u^{\prime}-z^{\prime}, u_{3}\right), g=\left(f^{\prime}+\Delta z^{\prime}, f_{3}\right)$ in $\Omega$ and extending $v, p, g, z^{\prime}$ to $\omega \times(-1,1)$ by taking

$$
\begin{array}{ccc}
v^{\prime}\left(x^{\prime}, x_{3}\right)=v^{\prime}\left(x^{\prime},-x_{3}\right), & v_{3}\left(x^{\prime}, x_{3}\right)=-v_{3}\left(x^{\prime},-x_{3}\right), \quad p\left(x^{\prime}, x_{3}\right)=p\left(x^{\prime},-x_{3}\right), \\
g^{\prime}\left(x^{\prime}, x_{3}\right)=g^{\prime}\left(x^{\prime},-x_{3}\right), \quad g_{3}\left(x^{\prime}, x_{3}\right)=-g_{3}\left(x^{\prime},-x_{3}\right), \quad z^{\prime}\left(x^{\prime}, x_{3}\right)=z^{\prime}\left(x^{\prime},-x_{3}\right),
\end{array}
$$

for $x^{\prime} \in \omega, x_{3} \in(-1,0)$, we deduce that $(v, p)$ satisfies

$$
\left\{\begin{array}{l}
-\Delta v+\nabla p=g \text { in } \omega \times(-1,1), \quad \operatorname{div} v=-\operatorname{div}_{x^{\prime}} z^{\prime} \text { in } \omega \times(-1,1) \\
v=0 \text { on } \omega \times\{-1,1\}, \quad \int_{\omega \times(-1,1)} p d x=0 .
\end{array}\right.
$$

Classical estimates for the Stokes problem with Dirichlet conditions (see e.g. [19]) show then the result.

The complete proofs of Theorems 1 and 2 and their generalizations to the NavierStokes system appear in [11]. For the proofs of Theorem 3 and Proposition 4 we refer to [13].

\section{Asymptotic behavior of viscous fluids in rough domains with small height}

In this section we consider the case of a viscous fluid confined in the thin rough domain $\Omega_{\varepsilon}^{\text {thin }}$ defined by (9). Remark that $\Omega_{\varepsilon}^{\text {thin }}$ has a rough bottom which agrees with $\Gamma_{\varepsilon}$ defined by (7). We still denote $\Omega=\omega \times(0,1)$ and $\Gamma=\omega \times\{0\}$.

Analogously to Section 2, we consider the Stokes system in $\Omega_{\varepsilon}^{\text {thin }}$ together with the Navier boundary condition on $\Gamma_{\varepsilon}$ and the adherence condition on the rest of the boundary $\partial \Omega_{\varepsilon}^{\text {thin }} \backslash \Gamma_{\varepsilon}$, that is

$$
\left\{\begin{array}{l}
-\Delta u_{\varepsilon}+\nabla p_{\varepsilon}=f \text { in } \Omega_{\varepsilon}^{\text {thin }}, \quad \operatorname{div} \mathrm{u}_{\varepsilon}=0 \text { in } \Omega_{\varepsilon}^{\text {thin }}, \\
u_{\varepsilon} \cdot \nu=0 \text { on } \Gamma_{\varepsilon}, \quad T_{\varepsilon}\left(\frac{\partial u_{\varepsilon}}{\partial \nu}+\frac{\gamma}{h_{\varepsilon}} u_{\varepsilon}\right)=0 \text { on } \Gamma_{\varepsilon} \\
u_{\varepsilon}=0 \text { on } \partial \Omega_{\varepsilon}^{\text {thin }} \backslash \Gamma_{\varepsilon},
\end{array}\right.
$$


where $\gamma \geq 0$ is a friction coefficient, $\nu$ denotes the unitary outside normal vector to $\Omega_{\varepsilon}^{\text {thin }}$ on $\bar{\Gamma}_{\varepsilon}, T_{\varepsilon}$ is the orthogonal projection on the tangent space to $\Gamma_{\varepsilon}$ and the second member $f$ belongs to $C\left(\mathbb{R} ; L^{2}(\omega)\right)^{3}$ (more general second members can be considered).

The system (24) has a unique solution $\left(u_{\varepsilon}, p_{\varepsilon}\right) \in H^{1}\left(\Omega_{\varepsilon}^{\text {thin }}\right)^{3} \times L_{0}^{2}\left(\Omega_{\varepsilon}^{\text {thin }}\right)$. Moreover, the following estimates hold

$$
\begin{gathered}
\frac{1}{\left|\Omega_{\varepsilon}^{\text {thin }}\right|} \int_{\Omega_{\varepsilon}^{\text {thin }}}\left|u_{\varepsilon}\right|^{2} d x \leq C h_{\varepsilon}^{4}, \quad \frac{1}{\left|\Omega_{\varepsilon}^{\text {thin }}\right|} \int_{\Omega_{\varepsilon}^{\text {thin }}}\left|D u_{\varepsilon}\right|^{2} d x \leq C h_{\varepsilon}^{2}, \\
\frac{1}{\left|\Omega_{\varepsilon}^{\text {thin }}\right|} \int_{\Omega_{\varepsilon}^{\text {thin }}}\left|p_{\varepsilon}\right|^{2} d x \leq C, \quad \forall \varepsilon>0 .
\end{gathered}
$$

Remark 3 The proof of estimates (25) for $u_{\varepsilon}$ and $p_{\varepsilon}$ easyly follows taking $u_{\varepsilon}$ as test function in (24) and then using the inequalities

$$
\begin{gathered}
\|v\|_{L^{2}\left(\Omega_{\varepsilon}^{t h i n}\right)} \leq C h_{\varepsilon}\|\nabla v\|_{L^{2}\left(\Omega_{\varepsilon}^{t h i n}\right)^{3}}, \quad \forall v \in H^{1}\left(\Omega_{\varepsilon}\right), v=0 \text { on } x_{3}=h_{\varepsilon} . \\
\|q\|_{L^{2}\left(\Omega_{\varepsilon}^{t h i n}\right)} \leq \frac{C}{h_{\varepsilon}}\|\nabla q\|_{H^{-1}\left(\Omega_{\varepsilon}^{t h i n}\right)^{3}}, \quad \forall q \in L^{2}\left(\Omega_{\varepsilon}\right) \text { with } \int_{\Omega_{\varepsilon}} q d x=0 .
\end{gathered}
$$

Our goal is to study the asymptotic behavior of $u_{\varepsilon}$ and $p_{\varepsilon}$ when $\varepsilon$ tends to zero. For this purpose, as usual, we use a dilatation in the variable $x_{3}$ to have functions defined in an open set of fixed height. Namely, we define $\tilde{u}_{\varepsilon} \in H^{1}(\Omega)^{3}, \tilde{p}_{\varepsilon} \in L_{0}^{2}(\Omega)$ by

$$
\tilde{u}_{\varepsilon}(y)=u_{\varepsilon}\left(y^{\prime}, h_{\varepsilon} y_{3}\right), \quad \tilde{p}_{\varepsilon}(y)=p_{\varepsilon}\left(y^{\prime}, h_{\varepsilon} y_{3}\right), \quad \text { a.e. } y \in \Omega .
$$

Then, the problem becomes in studying the asymptotic behavior of the functions $\tilde{u}_{\varepsilon}$ and $\tilde{p}_{\varepsilon}$. This is given by

Theorem 5 Let $\left(u_{\varepsilon}, p_{\varepsilon}\right) \in H^{1}\left(\Omega_{\varepsilon}^{\text {thin }}\right)^{3} \times L_{0}^{2}\left(\Omega_{\varepsilon}^{\text {thin }}\right)$ be the solution of the Stokes system (24) and let $\tilde{u}_{\varepsilon}, \tilde{p}_{\varepsilon}$ be defined by (26). Then, there exist $v^{\prime} \in H^{1}\left(0,1 ; L^{2}(\omega)\right)^{2}$, $w \in H^{2}\left(0,1 ; H^{-1}(\omega)\right)$ and $p \in H^{1}(\omega)$, with null integral, such that

$$
\begin{gathered}
\frac{\tilde{u}_{\varepsilon}}{h_{\varepsilon}} \rightarrow 0 \text { in } H^{1}(\Omega)^{3}, \frac{\tilde{u}_{\varepsilon}^{\prime}}{h_{\varepsilon}^{2}} \rightarrow v^{\prime} \text { in } H^{1}\left(0,1 ; L^{2}(\omega)\right)^{2}, \\
\frac{\tilde{u}_{\varepsilon, 3}}{h_{\varepsilon}^{3}} \rightarrow w \text { in } H^{2}\left(0,1 ; H^{-1}(\omega)\right), \\
\tilde{p}_{\varepsilon} \rightarrow p \text { in } L^{2}(\Omega),
\end{gathered}
$$

where the functions $v^{\prime}, w$ and p satisfy the following simplified Stokes system

$$
\left\{\begin{array}{l}
-\partial_{y_{3} y_{3}}^{2} v^{\prime}+\nabla_{y^{\prime}} p=f^{\prime}\left(y^{\prime}, 0\right) \quad \text { in } \Omega \\
\operatorname{div}_{\mathrm{y}^{\prime} \mathrm{v}^{\prime}}+\partial_{\mathrm{y}_{3}} \mathrm{w}=0 \quad \text { in } \Omega \\
\int_{0}^{1} v^{\prime}\left(y^{\prime}, y_{3}\right) d y_{3} \cdot \nu=0 \quad \text { on } \partial \omega \\
w\left(y^{\prime}, 0\right)=w\left(y^{\prime}, 1\right)=0 \quad \text { in } \omega, \quad v^{\prime}\left(y^{\prime}, 1\right)=0 \quad \text { in } \omega .
\end{array}\right.
$$


Moreover, according to the value of $\lambda_{\text {thin }}$ defined by (11), $v^{\prime}$ satisfies the following boundary condition on $\Gamma$ :

i) If $\lambda_{\text {thin }}=0$, then we have

$$
-\partial_{3} v^{\prime}+\gamma v^{\prime}=0, \quad \text { on } \Gamma .
$$

ii) If $\lambda_{\text {thin }} \in(0,+\infty)$, then we have

$$
-\partial_{3} v^{\prime}+\gamma v^{\prime}+\lambda_{\text {thin }}^{2} R v^{\prime}=0, \quad \text { on } \Gamma,
$$

where $R$ is defined by (17).

iii) If $\lambda_{\text {thin }}=+\infty$, then we have

$$
-\partial_{3} v^{\prime}+\gamma v^{\prime} \in W, \quad v^{\prime} \in W^{\perp}, \quad \text { on } \Gamma,
$$

where $W$ is defined by (19).

Sketch of the proof. Since $\left(u_{\varepsilon}, p_{\varepsilon}\right)$ satisfies (25), and $\operatorname{div} u_{\varepsilon}=0$ in $\Omega_{\varepsilon}$, we get the estimates

$$
\begin{gathered}
\int_{\Omega}\left|\frac{\tilde{u}_{\varepsilon}}{h_{\varepsilon}^{2}}\right|^{2} d y \leq C, \quad \int_{\Omega}\left(\left|D_{y^{\prime}}\left(\frac{\tilde{u}_{\varepsilon}}{h_{\varepsilon}}\right)\right|^{2}+\left|\partial_{y_{3}}\left(\frac{\tilde{u}_{\varepsilon}}{h_{\varepsilon}^{2}}\right)\right|^{2}\right) d y \leq C, \\
\int_{\Omega}\left|\tilde{p}_{\varepsilon}\right|^{2} d y \leq C, \quad \operatorname{div}_{y^{\prime}}\left(\frac{\tilde{u}_{\varepsilon}^{\prime}}{h_{\varepsilon}^{2}}\right)+\partial_{y_{3}}\left(\frac{\tilde{u}_{\varepsilon, 3}}{h_{\varepsilon}^{3}}\right)=0 \text { in } \Omega,
\end{gathered}
$$

for every $\varepsilon>0$. This implies the existence of $v^{\prime} \in H^{1}\left(0,1 ; L^{2}(\omega)\right)^{2}, w \in$ $H^{2}\left(0,1 ; H^{-1}(\omega)\right)$ and $p \in H^{1}(\omega)$, such that (27) holds. Then, taking into account that $\tilde{u}_{\varepsilon}=0$ on $\partial \Omega \backslash \Gamma$, and using as test function in (24) a sequence of the form $z_{\varepsilon}=h_{\varepsilon}^{2}\left(v\left(x^{\prime}, x_{3} / \varepsilon\right), 0\right)$ with $v^{\prime}$ smooth and vanishing on $\partial \Omega$, we easily get (28). In order to finish the proof of Theorem 5 it only remains to obtain the boundary condition satisfied by $\tilde{v}^{\prime}$ on $\Gamma$. This follows reasoning similarly to the proof of Theorem 1 by introducing the sequence $\hat{u}_{\varepsilon}: \hat{\omega}_{\varepsilon} \times K_{\varepsilon} \rightarrow \mathbb{R}^{3}$ as

$$
\hat{u}_{\varepsilon}\left(x^{\prime}, z\right)=\sum_{\substack{k^{\prime} \in \mathbb{Z}^{2} \\ \varepsilon k^{\prime}+\varepsilon \mathbb{Z}^{\prime} \subset \omega}} u_{\varepsilon}\left(\varepsilon k^{\prime}+\varepsilon z^{\prime}, \varepsilon z_{3}\right) \chi_{\varepsilon k^{\prime}+\varepsilon Z^{\prime}}\left(x^{\prime}\right)
$$

with

$$
\hat{\omega}_{\varepsilon}=\bigcup_{\substack{k^{\prime} \in \mathbb{Z}^{2} \\ \varepsilon k^{\prime}+\varepsilon Z^{\prime} \subset \omega}}\left(\varepsilon k^{\prime}+\varepsilon Z^{\prime}\right), \quad K_{\varepsilon}=\left\{z \in Z^{\prime} \times \mathbb{R}:-\frac{\delta_{\varepsilon}}{\varepsilon} \Psi\left(z^{\prime}\right)<z_{3}<\frac{h_{\varepsilon}}{\varepsilon}\right\} .
$$

We remark that $p$ only depends on the horizontal variables, i.e. $p=p\left(x^{\prime}\right)$. From (28), (29), (30) and (31), as usual in the asymptotic study of fluids in thin domains, we can obtain a Reynolds problem for $p$. Indeed, we have the following result 
Corollary 6 Let $\left(u_{\varepsilon}, p_{\varepsilon}\right) \in H^{1}\left(\Omega_{\varepsilon}^{\text {thin }}\right)^{3} \times L_{0}^{2}\left(\Omega_{\varepsilon}^{\text {thin }}\right)$ be the solution of the Stokes system (24). Then, depending on the value of $\lambda_{\text {thin }}$ defined by (11), the functions $v^{\prime}, w$ and $p$ in Theorem 5 are given by

(i) If $\lambda_{\text {thin }}=0, p$ is the solution of the Reynolds problem

$$
\left\{\begin{array}{l}
-\operatorname{div}_{y^{\prime}}\left(\left(\frac{1}{3}+(1+\gamma)^{-1}\right)\left(\nabla_{y^{\prime}} p-f^{\prime}\left(y^{\prime}, 0\right)\right)\right)=0 \text { in } \omega \\
\left(\left(\frac{1}{3}+(1+\gamma)^{-1}\right)\left(\nabla_{y^{\prime}} p-f^{\prime}\left(y^{\prime}, 0\right)\right)\right) \cdot \nu=0 \text { on } \partial \omega
\end{array}\right.
$$

the function $v^{\prime}$ is given by

$$
v^{\prime}(y)=\frac{1}{2}\left(y_{3}^{2}+(1+\gamma)^{-1}\right)\left(\nabla_{y^{\prime}} p\left(y^{\prime}\right)-f^{\prime}\left(y^{\prime}, 0\right)\right), \quad \text { a.e. } y \in \Omega,
$$

and the distribution $w$ is zero.

(ii) If $\lambda_{\text {thin }} \in(0,+\infty)$, then defining $R$ by (17), we have that $p$ satisfies the following Reynolds problem

$$
\left\{\begin{array}{l}
-\operatorname{div}_{y^{\prime}}\left(\left(\frac{1}{3} I+\left((1+\gamma) I+\lambda_{\text {thin }}^{2} R\right)^{-1}\right)\left(\nabla_{y^{\prime}} p-f^{\prime}\left(y^{\prime}, 0\right)\right)\right)=0 \text { in } \omega \\
\left(\left(\frac{1}{3} I+\left((1+\gamma) I+\lambda_{\text {thin }}^{2} R\right)^{-1}\right)\left(\nabla_{y^{\prime}} p-f^{\prime}\left(y^{\prime}, 0\right)\right)\right) \cdot \nu=0 \text { on } \partial \omega
\end{array}\right.
$$

the function $v^{\prime}$ is given by

$$
v^{\prime}(y)=\frac{\left(y_{3}-1\right)}{2 \mu}\left(y_{3} I+\left((1+\gamma) I+\lambda_{\text {thin }}^{2} R\right)^{-1}\right)\left(\nabla_{y^{\prime}} p\left(y^{\prime}\right)-f^{\prime}\left(y^{\prime}, 0\right)\right),
$$

a.e. $y \in \Omega$, and the distribution $w$ is given by

$$
w(y)=-\int_{0}^{y_{3}} \operatorname{div}_{\mathrm{y}^{\prime}} \mathrm{v}\left(\mathrm{y}^{\prime}, \mathrm{s}\right) \mathrm{ds} \text { in } \Omega .
$$

(iii) If $\lambda_{\text {thin }}=+\infty$, then denoting by $P_{W^{\perp}}$ the orthogonal projection from $\mathbb{R}^{2}$ to the orthogonal space of $W$ defined by (19), we have that $p$ is given as the solution of the Reynolds problem

$$
\left\{\begin{array}{l}
-\operatorname{div}_{y^{\prime}}\left(\left(\frac{1}{3} I+(1+\gamma)^{-1} P_{W^{\perp}}\right)\left(\nabla_{y^{\prime}} p-f^{\prime}\left(y^{\prime}, 0\right)\right)\right)=0 \text { in } \omega \\
\left(\left(\frac{1}{3} I+(1+\gamma)^{-1} P_{W^{\perp}}\right)\left(\nabla_{y^{\prime}} p-f^{\prime}\left(y^{\prime}, 0\right)\right)\right) \cdot \nu=0 \text { on } \partial \omega,
\end{array}\right.
$$

the function $v^{\prime}$ is given by

$$
v^{\prime}(y)=\frac{\left(y_{3}-1\right)}{2}\left(y_{3} I+(1+\gamma)^{-1} P_{W^{\perp}}\right)\left(\nabla_{y^{\prime}} p\left(y^{\prime}\right)-f^{\prime}\left(y^{\prime}, 0\right)\right),
$$

a.e. $y \in \Omega$, and the distribution $w$ by (33). 
Remark 4 The parameter $\lambda_{\text {thin }}$ in Theorem 5 plays a similar role to that of $\lambda$ defined in Theorem 1, i.e. if $\lambda_{\text {thin }}=0$ the roughness is too slight and it has no effect on the solution. If $\lambda_{\text {thin }}=+\infty$, the roughness is so strong that, in $\Gamma$, $v^{\prime}$ belongs to the orthogonal space of $W$ defined by (19). The case $\lambda_{\text {thin }} \in(0,+\infty)$ is the critical case where the roughness is not strong enough to imply $v^{\prime} \in W^{\perp}$ in the limit, but it is enough to produce a new friction term $\lambda_{\text {thin }}^{2} R v^{\prime}$, where $R$ is the matrix obtained in Theorem 1 .

Remark 5 We remark that taking $h_{\varepsilon}=1$ in (11), the parameters $\lambda$ and $\lambda_{\text {thin }}$ agree. In the case of thin domains, the expression of $\lambda_{\text {thin }}$ does not only depend on the parameters $\delta_{\varepsilon}, \varepsilon$ which define $\Gamma_{\varepsilon}$, but also on the height $h_{\varepsilon}$ of $\Omega_{\varepsilon}^{\text {thin }}$. This is due to the fact that far of the rough boundary the behavior of the fluid is different from the corresponding one in Section 2.

Finally, we give corrector results for the velocity and the pressure in the following theorem.

Theorem 7 Assume $\omega \in C^{2}$ and $f\left(x^{\prime}, 0\right) \in H^{1}(\omega)^{2}$. Let $\left(u_{\varepsilon}, p_{\varepsilon}\right) \in H^{1}\left(\Omega_{\varepsilon}^{\text {thin }}\right)^{3} \times$ $L_{0}^{2}\left(\Omega_{\varepsilon}^{\text {thin }}\right)$ be the solution of the Stokes system (24). Then we have

i) If $\lambda_{\text {thin }}=0$ or $+\infty$, defining $\breve{u}_{\varepsilon}$ by

$$
\breve{u}_{\varepsilon}(x)=\left(h_{\varepsilon}^{2} v^{\prime}\left(x^{\prime}, \frac{x_{3}}{h_{\varepsilon}}\right), 0\right), \text { a.e. } x \in \Omega_{\varepsilon}^{\text {thin }},
$$

we have

$$
\begin{gathered}
\frac{1}{h_{\varepsilon}^{5}} \int_{\Omega_{\varepsilon}^{t h i n}}\left|u_{\varepsilon}-\breve{u}_{\varepsilon}\right|^{2} d x \rightarrow 0, \quad \frac{1}{h_{\varepsilon}^{3}} \int_{\Omega_{\varepsilon}^{t h i n}}\left|D\left(u_{\varepsilon}-\breve{u}_{\varepsilon}\right)\right|^{2} d x \rightarrow 0, \\
\frac{1}{h_{\varepsilon}} \int_{\Omega_{\varepsilon}^{t h i n}}\left|p_{\varepsilon}-p\right|^{2} d x \rightarrow 0 .
\end{gathered}
$$

ii) If $\lambda_{\text {thin }} \in(0,+\infty)$, the above assertions still hold replacing $\breve{u}_{\varepsilon}$ by

$$
\breve{u}_{\varepsilon}(x)=\left(h_{\varepsilon}^{2} v^{\prime}\left(x^{\prime}, \frac{x_{3}}{h_{\varepsilon}}\right), 0\right)+\lambda_{t h i n} h_{\varepsilon}^{\frac{3}{2}} \sqrt{\varepsilon}\left(v_{1}^{\prime}\left(x^{\prime}, 0\right) \widehat{\phi}^{1}\left(\frac{x}{\varepsilon}\right)+v_{2}^{\prime}\left(x^{\prime}, 0\right) \widehat{\phi}^{2}\left(\frac{x}{\varepsilon}\right)\right),
$$

with $\widehat{\phi}^{i}, i=1,2$, the solutions of (16).

The results given in this section were announce in [12]. The generalization of these results to Navier-Stokes system will appear in a forthcoming paper [14].

\section{Asymptotic behavior of elliptic systems in general rough domains}

In the previous sections we have shown that the Navier boundary condition for the Stokes system provides a new term in the limit problem. In this section we study this phenomena for linear elliptic systems in rough domains $\Omega_{\varepsilon} \subset \mathbb{R}^{N}$, where $\Omega_{\varepsilon}$ has not 
necessarily a periodic structure.

We consider a sequence of Lipschitz open sets $\Omega_{\varepsilon} \subset \mathbb{R}^{N}$ which converges to a Lipschitz open set $\Omega \subset \mathbb{R}^{N}$ in the following sense: For every $\rho>0$, there exists $\varepsilon_{0}>0$ such that for every $\varepsilon \in\left(0, \varepsilon_{0}\right)$, we have (see Figure 3 )

$$
\Omega^{\rho^{-}}=\{x \in \Omega: d(x, \partial \Omega)>\rho\} \subset \Omega_{\varepsilon} \subset\left\{x \in \mathbb{R}^{N}: d(x, \bar{\Omega})<\rho\right\}=\Omega^{\rho^{+}} .
$$

We denote by $\widetilde{\Omega}$ an open set containing completely $\Omega$.

In $\Omega_{\varepsilon}$, we consider the following homogenization problem

$$
\left\{\begin{array}{l}
-\operatorname{div} A D u_{\varepsilon}=f \quad \text { in } \Omega_{\varepsilon} \\
u_{\varepsilon}(x) \in V_{\varepsilon}(x), \quad \forall x \in \partial \Omega_{\varepsilon}, \quad A D u_{\varepsilon}(x) \cdot \nu \in V_{\varepsilon}(x)^{\perp} \quad \forall x \in \partial \Omega_{\varepsilon},
\end{array}\right.
$$

where $A$ belongs to $L^{\infty}\left(\widetilde{\Omega} ; \mathcal{T}_{M \times N}\right)\left(\mathcal{T}_{M \times N}\right.$ is the space of linear functions from the space of matrices $\mathcal{M}_{M \times N}$ into itself), $V_{\varepsilon}(x)$ is an arbitrary sequence of functions from $\partial \Omega_{\varepsilon}$ into the set of linear subspaces of $\mathbb{R}^{M}$, and the second member $f$ is a function in $L^{2}(\widetilde{\Omega})^{M}$.

We also assume the following ellipticity condition: there exists $\alpha>0$ such that

$$
\left\{\begin{array}{l}
\alpha\|v\|_{H^{1}\left(\Omega_{\varepsilon}\right)^{M}}^{2} \leq \int_{\Omega_{\varepsilon}} A D v: D v d x \\
\forall v \in H^{1}\left(\Omega_{\varepsilon}\right)^{M}, v(x) \in V_{\varepsilon}(x), \text { a.e. } x \in \partial \Omega_{\varepsilon} .
\end{array}\right.
$$

Observe that this ellipticity condition is written in an integral form instead of in a pointwise one. This is more convenient for systems, where the pointwise and integral ellipticity conditions are not equivalent. In particular it permits to deal with the linear elasticity system, where the tensor only depends on the symmetric part of the derivative.

Assuming that $V_{\varepsilon}(x)=T_{\varepsilon}(x)$, with $T_{\varepsilon}(x)$ the tangent space in the point $x \in \partial \Omega_{\varepsilon}$, the oscillating boundary condition in (37) is similar to the Navier boundary condition (see (12)) considered in Sections 2 and 3. Some other choices of $V_{\varepsilon}$ are also interesting. For example, taking $S_{\varepsilon}$ an arbitrary subset of $\partial \Omega_{\varepsilon}$, and defining $V_{\varepsilon}$ as $V_{\varepsilon}(x)=\{0\}$ for $x \in S_{\varepsilon}$, and $V_{\varepsilon}(x)=\mathbb{R}^{N}$ for $x \in \partial \Omega_{\varepsilon} \backslash S_{\varepsilon}$, the homogenization problem reads

$$
\left\{\begin{array}{l}
-\operatorname{div} A D u_{\varepsilon}=f_{\varepsilon} \quad \text { in } \Omega_{\varepsilon} \\
u_{\varepsilon}=0 \quad \text { on } S_{\varepsilon}, \quad A D u_{\varepsilon}(x) \cdot \nu=0 \quad \text { on } \partial \Omega_{\varepsilon} \backslash S_{\varepsilon} .
\end{array}\right.
$$

In this case, we are studying the homogenization of elliptic partial systems with Dirichlet and Neumann conditions on varying subsets of the boundary. This problem has been studied in [7] and [8] in the particular case $\Omega_{\varepsilon}=\Omega$.

Our main result in this section is the following theorem 
Theorem 8 There exist a subsequence of $\varepsilon$, still denoted by $\varepsilon$, a Borel measure $\mu$ in $\partial \Omega$ which vanishes on the sets of null capacity, a $\mu$-measurable function $R: \partial \Omega \rightarrow$ $\mathcal{M}_{M \times M}$, with

$$
R \xi \cdot \xi \geq 0,|R \xi \cdot \eta| \leq \beta(R \xi \cdot \xi)^{\frac{1}{2}}(R \eta \cdot \eta)^{\frac{1}{2}}, \quad \forall \xi, \eta \in \mathbb{R}^{M}, \text { - } \text {-a.e. in } \partial \Omega,
$$

for some $\beta>0$, and a function $V$ from $\partial \Omega$ into the set of linear subspaces of $\mathbb{R}^{M}$, satisfying

$$
\begin{gathered}
\alpha\|v\|_{H^{1}(\Omega)^{M}}^{2} \leq \int_{\Omega} A D v: D v d x+\int_{\partial \Omega} R v \cdot v d \mu \\
\forall v \in H^{1}(\Omega)^{M}, \quad v \in V \text { q.e. on } \partial \Omega,
\end{gathered}
$$

with the following property: For every $f \in L^{2}(\widetilde{\Omega})^{M}$, the unique solution of (37) converges weakly in $H^{1}\left(\Omega^{\rho^{-}}\right)$, for every $\rho>0$, to the unique solution $u \in H^{1}(\Omega)^{M}$ of problem

$$
\left\{\begin{array}{l}
-\operatorname{div} A D u=f \quad \text { in } \Omega \\
u \in V, \quad \text { q.e. in } \partial \Omega, \quad \int_{\partial \Omega} R u \cdot u d \mu<+\infty \\
A D u \cdot \nu+R u \mu \in V^{\perp}, \quad \text { q.e. in } \partial \Omega .
\end{array}\right.
$$

Remark 6 The solution of (39) is understood as the solution of the following variational problem,

$$
\left\{\begin{array}{l}
u \in H^{1}(\Omega)^{M}, u(x) \in V(x) \text { q.e. in } \partial \Omega, \int_{\partial \Omega} R u \cdot u d \mu<+\infty \\
\int_{\Omega} A D u: D v d x+\int_{\partial \Omega} R u \cdot v d \mu=\int_{\Omega} f \cdot v d x \\
\forall v \in H^{1}(\Omega)^{M}, v(x) \in V(x) \text { q.e. in } \partial \Omega, \int_{\partial \Omega} R v \cdot v d \mu<+\infty .
\end{array}\right.
$$

Remark 7 Theorem 8 applies for instance to study the behavior of the elasticity system

$$
\left\{\begin{array}{l}
u_{\varepsilon} \in H^{1}\left(\Omega_{\varepsilon}\right)^{N}, \quad u_{\varepsilon} \in V_{\varepsilon}(x) \text { q.e. in } \partial \Omega_{\varepsilon} \\
\int_{\Omega_{\varepsilon}} B e\left(u_{\varepsilon}\right): e(v) d x=\int_{\Omega_{\varepsilon}} f \cdot v d x \\
\forall v \in H^{1}\left(\Omega_{\varepsilon}\right)^{N}, \quad v \in V_{\varepsilon}(x) \text { q.e. in } \partial \Omega_{\varepsilon}
\end{array}\right.
$$

where the rough domain $\Omega_{\varepsilon}$ is described by

$$
\Omega_{\varepsilon}=\left\{x=\left(x^{\prime}, x_{N}\right) \in \mathbb{R}^{N}: x^{\prime} \in \omega, \quad \Phi_{\varepsilon}\left(x^{\prime}\right) \leq x_{N} \leq 1\right\},
$$

with $\omega \subset \mathbb{R}^{N-1}$ is a Lipschitz open set and $\Phi_{\varepsilon}$ converging *-weakly to zero in $W^{1, \infty}(\omega)$.

Here, $\Omega=\omega \times(0,1), B \in L^{\infty}\left(\widetilde{\Omega} ; \mathcal{T}_{N, s}\right)\left(\mathcal{T}_{N, s}\right.$ is the space of linear applications from the space of symmetric matrices of order $N \times N, \mathcal{M}_{N, s}$, into itself) is such that there exists $\alpha>0$ satisfying

$$
B(x) \xi: \xi \geq \alpha|\xi|^{2}, \quad \forall \xi \in \mathcal{M}_{N, s}, \text { a.e. } x \in \partial \Omega,
$$


and $V_{\varepsilon}$ is a sequence of applications from $\partial \Omega_{\varepsilon}$ into the set of linear subspaces of $\mathbb{R}^{N}$ such that $V_{\varepsilon}\left(x^{\prime}, 1\right)=\{0\}$ for every $x^{\prime} \in \omega$.

Remark 8 Theorem 8 can also be extended to the Stokes system. In this case, taking $V_{\varepsilon}(x)=T_{\varepsilon}(x)$, we would recover the results in [6].

Sketch of the proof of Theorem 8. The proof of this result is given in [15], where we also prove that problem (40) is stable by homogenization. The idea is the following one:

Thanks to assumption (38), the norm of the solution $u_{\varepsilon}$ of problem (37) in $H^{1}\left(\Omega_{\varepsilon}\right)^{M}$ is bounded. Then, by a diagonal procedure, we can extract a subsequence of $\varepsilon$, still denoted by $\varepsilon$, such that for every $f \in L^{2}(\tilde{\Omega})^{M}$, there exists $u \in H^{1}(\Omega)^{M}$, such that the solution $u_{\varepsilon}$ of (37) converges weakly to $u$ in $H^{1}\left(\Omega^{\rho^{-}}\right)^{M}$, for every $\rho>0$. We define $W \subset H^{1}(\Omega)^{M}$ as the space of functions $u \in H^{1}(\Omega)^{M}$ which are the limit in the previous sense of a sequence $u_{\varepsilon}$ solution of (37), for some $f \in L^{2}(\tilde{\Omega})^{M}$. We consider $u^{1}, u^{2} \in W$, and sequences $u_{\varepsilon}^{1}, u_{\varepsilon}^{2}$ solutions of (37) with $f$ replaced by some $f^{1}, f^{2} \in L^{2}(\tilde{\Omega})^{M}$, such that $u_{\varepsilon}^{i}$ converges weakly to $u^{i}$ in $H^{1}\left(\Omega^{\rho^{-}}\right)^{M}$, for every $\rho>0, i=1,2$. For $\varphi \in C^{1}(\tilde{\Omega})$, we take $u_{\varepsilon}^{2} \varphi$ as test function in the equation for $u_{\varepsilon}^{1}$. Then, passing to the limit, we easily get

$$
\int_{\Omega} A D u^{1}: D\left(u^{2} \varphi\right) d x+\lim _{\varepsilon \rightarrow 0} \int_{\Omega_{\varepsilon}} A D\left(u_{\varepsilon}^{1}-u^{1}\right): D\left(u_{\varepsilon}^{2}-u^{2}\right) \varphi d x=\int_{\Omega} f^{1} u^{2} \varphi d x .
$$

The conclusion of Theorem 8 follows proving that the bilinear function $\nu$ from $W \times W$ into the space of Radon measures in $\bar{\Omega}$, defined as

$$
\int_{\bar{\Omega}} \varphi d \nu\left(u^{1}, u^{2}\right)=\lim _{\varepsilon \rightarrow 0} \int_{\Omega_{\varepsilon}} A D\left(u_{\varepsilon}^{1}-u^{1}\right): D\left(u_{\varepsilon}^{2}-u^{2}\right) \varphi d x
$$

is in the conditions of Theorem 4.1 in [15] (which is a variant of an integral representation result given in [18]). This proves the existence of $V, \mu$ an $R$ in the conditions of the statement of Theorem 8, such that $W$ is dense in the space of functions $u \in H^{1}(\Omega)^{M}$ with $R u \cdot u \in L_{\mu}^{1}(\partial \Omega)$ and such that

$$
\int_{\bar{\Omega}} \varphi d \nu\left(u^{1}, u^{2}\right)=\int_{\partial \Omega} R u^{1} \cdot u^{2} \varphi d \mu, \quad \forall u^{1}, u^{2} \in W, \forall \varphi \in C^{1}(\tilde{\Omega}) .
$$

This proves that $u^{1}$ satisfies

$\int_{\Omega} A D u^{1}: D\left(u^{2} \varphi\right) d x+\int_{\partial \Omega} R u^{1} \cdot u^{2} \varphi d \mu=\int_{\Omega} f^{1} u^{2} \varphi d x, \quad \forall u^{2} \in W, \forall \varphi \in C^{1}(\tilde{\Omega})$ and then that the limit $u$ of the solution $u_{\varepsilon}$ of (37) is the solution of (40).

\section{Acknowledgements}

The authors thank D. Bresch, E. Fernández-Cara and F. Murat for interesting discussions and many suggestions concerning this paper. This work have been partially supported by the project MTM2008-00306 of the "Ministerio de Ciencia e Innovación" and the Research group of the "Junta de Andalucía" FQM309. 


\section{Figures}

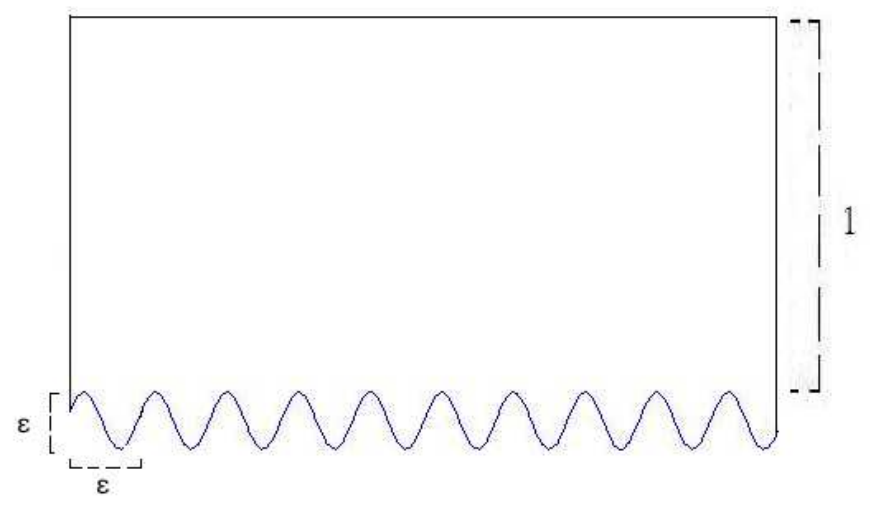

Figure 1: Rough domain $\Omega_{\varepsilon}$ with a rough bottom described by (4).

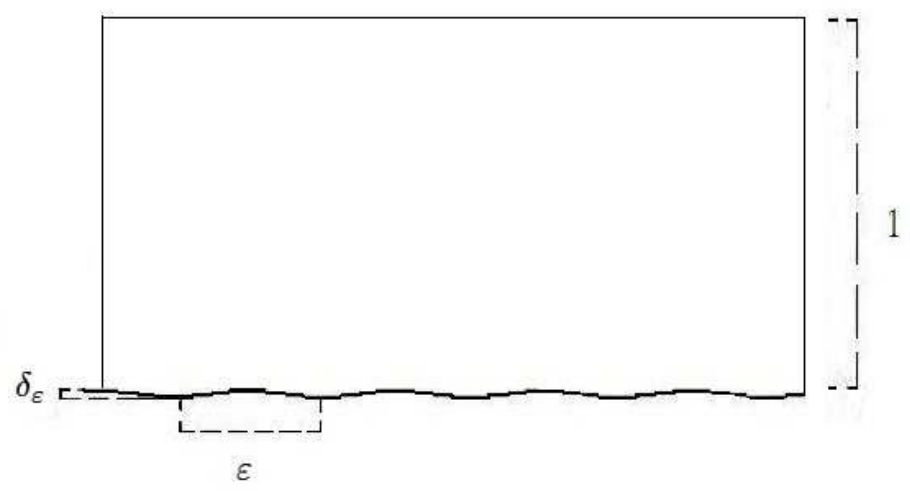

Figure 2: Rough domain $\Omega_{\varepsilon}$ described by (6). 


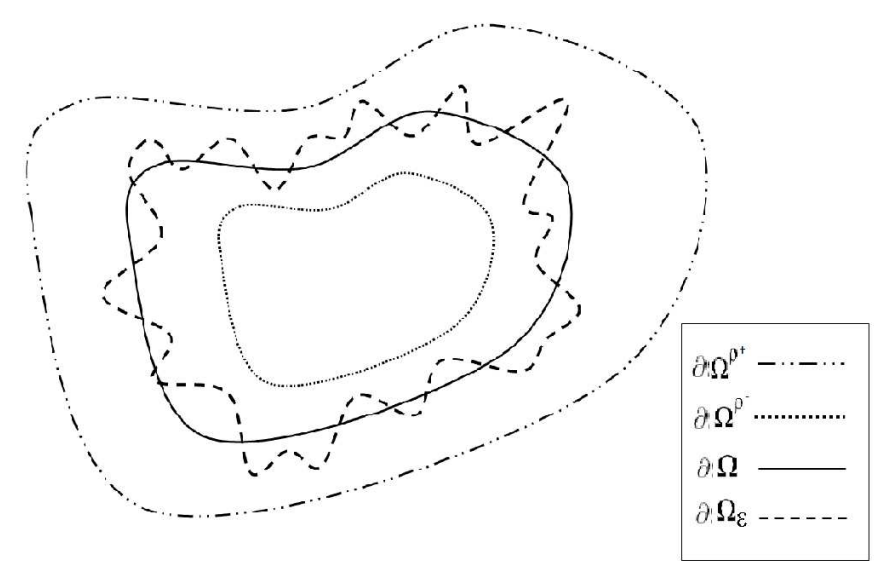

Figure 3: Convergence of the rough domain $\Omega_{\varepsilon}$ defined by (36).

\section{References}

[1] G. Allaire. Homogenization and two-scale convergence. SIAM J. Math. Anal., 23:1482-1518, 1992.

[2] T. Arbogast, J. Douglas, and U. Hornung. Derivation of the double porosity model of single phase flow via homogenization theory. SIAM J. Math. Anal., 21:823-836, 1990 .

[3] D. Bucur, E. Feireisl, S. Nečasová, and J. Wolf. On the asymptotic limit of the Navier-Stokes system on domains with rough boundaries. J. Differential Equations, 244: 2890-2908, 2008.

[4] D. Bucur, E. Feireisl, and S. Nečasová. Influence of wall roughness on the slip behavior of viscous fluids. Proc. Royal Soc. Edinburgh A, 138,5:957-973, 2008.

[5] D. Bucur, E. Feireisl, and S. Nečasová. On the asymptotic limit of flows past a ribbed boundary, J. Math. Fluid Mech., 10,4: 554-568, 2008.

[6] D. Bucur, E. Feireisl, and S. Nečasová. Boundary behavior of viscous fluids: Influence of wall roughness and friction-driven boundary conditions. Arch. Rational Mech. Anal., 197:117-138, 2010.

[7] C. Calvo-Jurado, J. Casado-Díaz, M. Luna-Laynez. Homogenization of elliptic problems with Dirichlet and Neumann conditions imposed on varying subsets. Math. Methods Appl. Sci. 30, 14:1611-1625, 2007.

[8] C. Calvo-Jurado, J. Casado-Díaz, M. Luna-Laynez. Asymptotic behavior of nonlinear systems in varying domains with boundary conditions on varying sets. ESAIM Control, Optim. and Calc. Var., 15: 49-67, 2009. 
[9] J. Casado-Díaz. Two-scale convergence for nonlinear Dirichlet problems in perforated domains. Proc. Roy. Soc. Edimburgh A, 130: 249-276, 2000.

[10] J. Casado-Díaz, E. Fernández-Cara, and J. Simon. Why viscous fluids adhere to rugose walls: A mathematical explanation. J. Differential Equations, 189, 2: 526-537, 2003.

[11] J. Casado-Díaz, M. Luna-Laynez, and F.J. Suárez-Grau. Asymptotic behavior of a viscous fluid with slip boundary conditions on a slightly rough wall. Math. Mod. Meth. Appl. Sci., 20: 121-156, 2010.

[12] J. Casado-Díaz, M. Luna-Laynez, and F.J. Suárez-Grau. A viscous fluid in a thin domain satisfying the slip condition on a slightly rough boundary. C. R. Acad. Sci. Paris, Ser. I, 348: 967-971, 2010.

[13] J. Casado-Díaz, M. Luna-Laynez, and F.J. Suárez-Grau. Estimates for the asymptotic expansion of a viscous fluid satisfying Navier's law on a rugous boundary. Math. Meth. Appl. Sci., 34: 1553-1561, 2011.

[14] J. Casado-Díaz, M. Luna-Laynez, and F.J. Suárez-Grau. Asymptotic behavior of the Navier-Stokes system in a thin domain with the slip condition on a slightly rough boundary, submitted for publication.

[15] J. Casado-Díaz, M. Luna-Laynez, and F.J. Suárez-Grau. The homogenization of elliptic partial differential systems on rugous domains with variable boundary conditions, to appear in Proc. Royal Soc. Edinburgh A.

[16] D. Cioranescu , A. Damlamian, and G. Griso, Periodic unfolding and homogenization. C. R. Acad. Sci. Paris, Sér. I, 335: 99-104, 2002.

[17] D. Cioranescu, and F. Murat. Un terme étrange venu d'ailleurs, Nonlinear partial differential equations and their applications. Collège de France seminar, Vols II and III. Eds H. Brézis and J. L. Lions. Research Notes in Math. 60 and 70. Pitman, London, 98-138 and 154-78, 1982.

[18] G. Dal Maso, A. Defranceschi, and E. Vitali. Integral representation for a class of $C^{1}$-convex functionals, J. Math. Pures Appl. 9, 73: 1-46, 1994.

[19] G.P. Galdi. An introduction to the mathematical theory of the Navier-Stokes equations. Vol. 1, Springer-Verlag, New-York, 1994.

[20] P. Grisvard. Elliptic problems in nonsmooth domains. Pitman, Boston, 1985.

[21] Lenczner M., Homogénéisation d'un circuit électrique, C. R. Acad. Sci. Paris II, 324,b: 537-542, 1997.

[22] C.L.M.H. Navier. Mémoire sur les lois du mouvement des fluides. Mem. Acad. R. Sci. Paris 6: 389-416, 1823.

[23] G. Nguetseng. A general convergence result for a functional related to the theory of homogenization. SIAM J. Math. Anal., 20, 3: 608-623, 1989. 
[24] F.J. Suárez-Grau. Comportamiento asintótico de fluidos viscosos con condiciones de deslizamiento sobre fronteras rugosas. Ph.D. Dissertation, Universidad de Sevilla, 2011. 